\title{
Coulomb effects in polarization transfer in elastic antiproton and proton electron scattering at low energies
}

\author{
H. Arenhövel ${ }^{\mathrm{a}}$ \\ Institut für Kernphysik, Johannes Gutenberg-Universität Mainz, D-55099 Mainz, Germany
}

Original article: Eur. Phys. J. A 34, 303 (2007) DOI: 10.1140/epja/i2007-10452-0

Received: 12 December 2008

Published online: 9 January 2009 - (C) Società Italiana di Fisica / Springer-Verlag 2009

In view of the criticism raised recently by Milstein et al. [1] our code for the numerical evaluation, based on an integral representation, has been checked against the analytic evaluation of [1] based on a partial-wave expansion. It turned out that, besides a minor error, the main reason for the overestimation of the polarisation transfer crosssection for elastic electron proton scattering at low energies is caused by the fact that for larger positive values of the Coulomb parameter $\eta(\eta \geq 1.5)$, corresponding to a proton kinetic energy $T_{h} \leq 0.01 \mathrm{MeV}$, the tensor $D_{i j}$ of the hyperfine interaction

$$
D_{i j}(\eta)=N(\eta)^{2} Q(\eta)^{2}\left(R_{i j}(\eta)+I_{i j}(\eta)\right),
$$

in eq. (A.30) of the appendix of [2] is given by the difference of two almost equal numbers $R_{i j}(\eta) \approx-I_{i j}(\eta)$ multiplied by a huge number $N(\eta)^{2} Q(\eta)^{2}$. This fact led numerically to the large overestimations for $T_{h} \leq 0.01 \mathrm{MeV}$, even with double precision $($ real*8).

Figures 1 and 2 (in the following pages) exhibit the corrected results for figs. 1 and 2 of [2]. For electron proton scattering one still notices an enhancement compared to the plane-wave approximation (PW) but of much smaller size. Thus the differential cross-section ratios with/without the hyperfine interaction shown in fig. 3 of [2] now are one everywhere. This means, that the DWBA is a good approximation for all energies considered.

Figures 3 and 4 (in the following pages) correspond to figs. 4 and 5 of [2] and display the integrated polarization transfer cross-sections for antiproton and proton electron scattering, respectively. Again one readily notices at energies below $0.01 \mathrm{MeV}$ a much smaller transfer cross-section.

Corresponding corrections apply to the theoretical results for the triple polarization cross-sections in [3] and will be presented in a separate erratum [4].

I would like to thank Th. Walcher for his help in checking the radial integrals.

\section{References}

1. A.I. Milstein, S.G. Salnikov, V.M. Strakhovenko, Nucl. Instrum. Methods B 266, 3453 (2008) [arXiv:0802.3766 [hep-ph]].

2. H. Arenhövel, Eur. Phys. J. A 34, 303 (2007).

3. Th. Walcher et al., Eur. Phys. J. A 34, 447 (2007).

4. Th. Walcher et al., Erratum to Eur. Phys. J. A 34, 447 (2007) (this issue).

\footnotetext{
${ }^{a}$ e-mail: arenhoev@kph.uni-mainz.de
} 

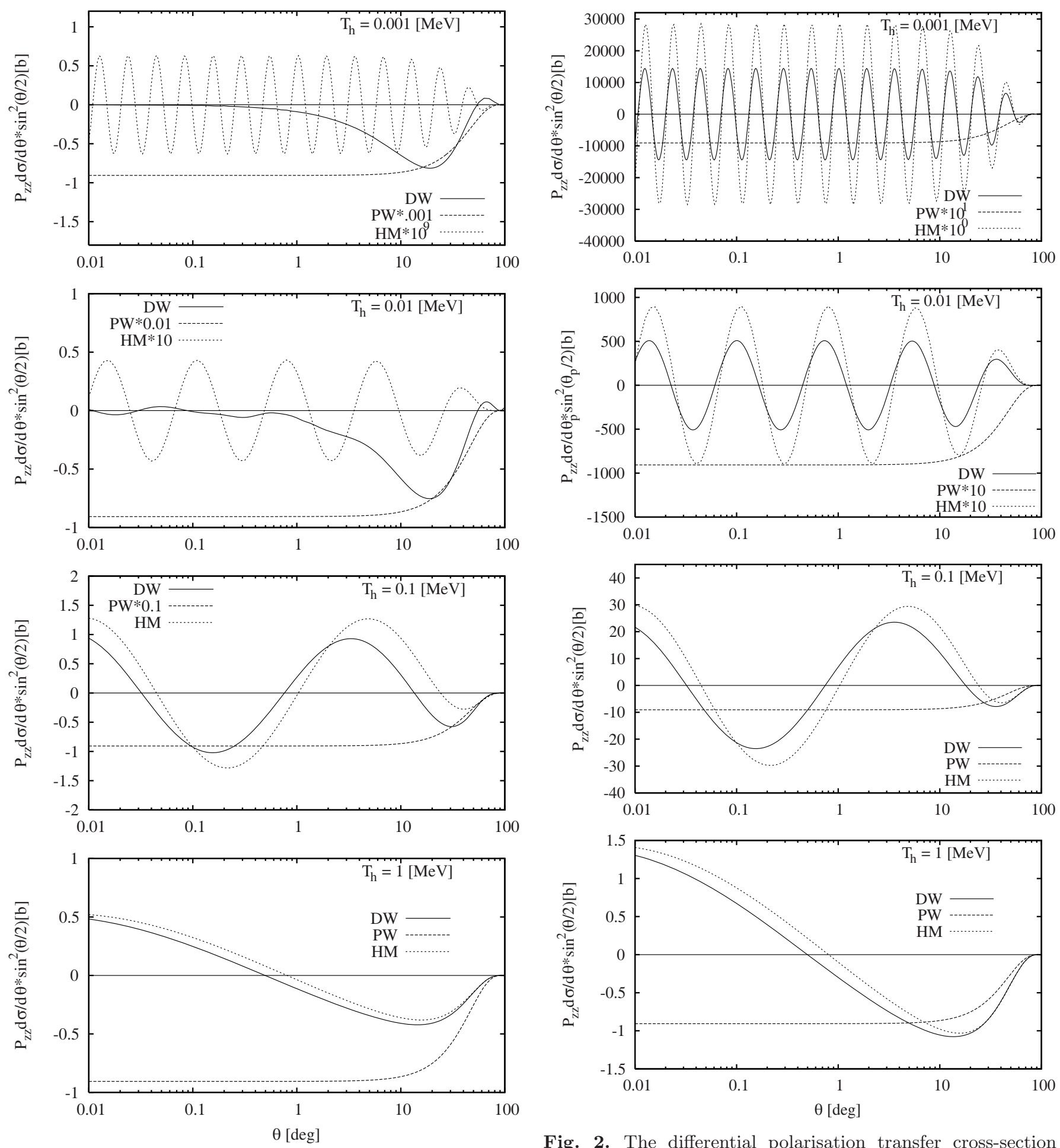

Fig. 1. The differential polarisation transfer cross-section weighted by $\sin ^{2}(\theta / 2)$ at $\phi=0$ for electron antiproton scattering in the c.m. frame for various antiproton lab kinetic energies

Fig. 2. The differential polarisation transfer cross-section weighted by $\sin ^{2}(\theta / 2)$ at $\phi=0$ for electron proton scattering in the c.m. frame for various proton lab kinetic energies and for the approximations PW, HM, and DW. and for the approximations PW, HM, and DW. 

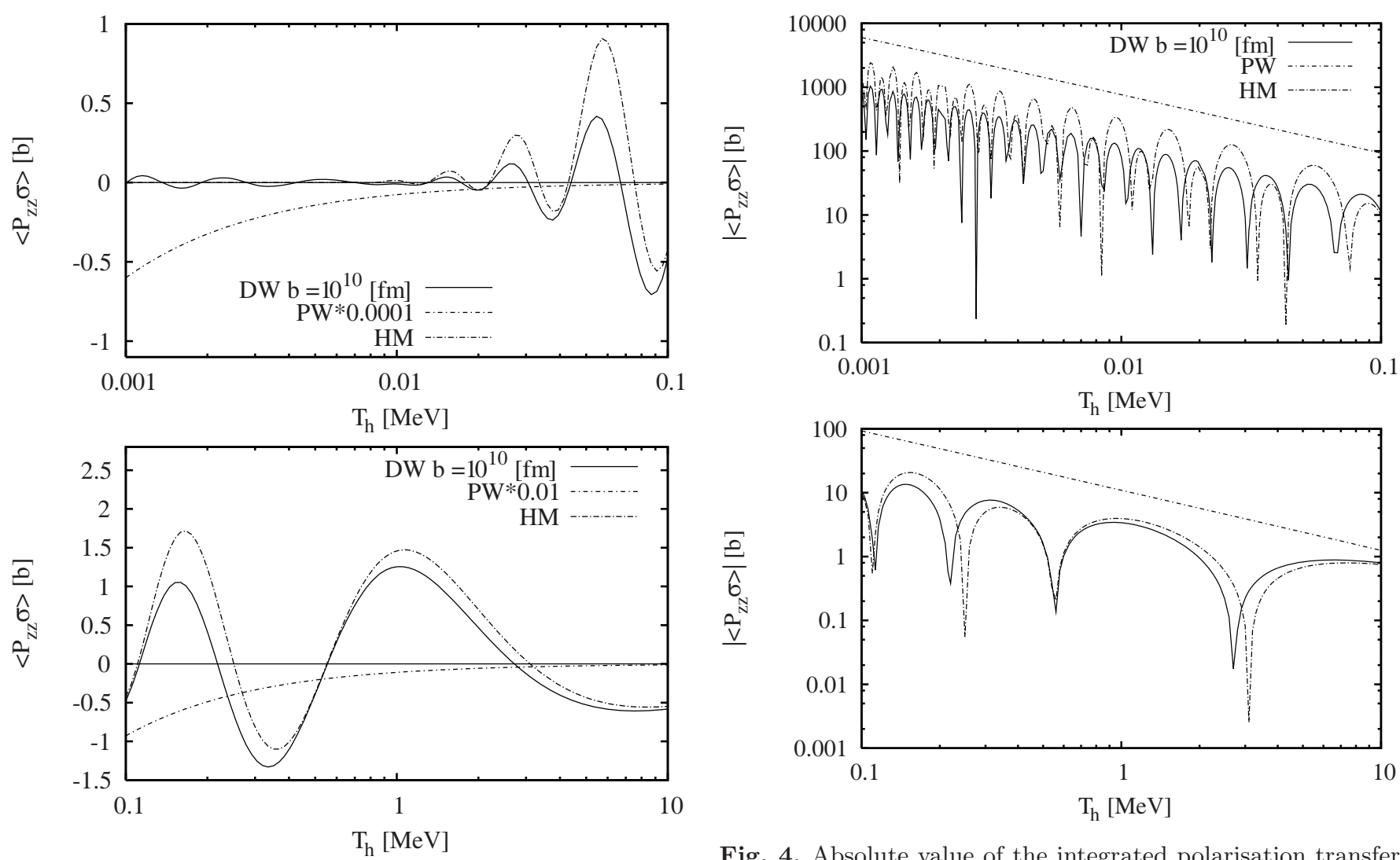

Fig. 3. The integrated polarisation transfer cross-section $\left\langle P_{z z} \sigma\right\rangle$ for antiproton electron scattering in the c.m. frame as a function of the antiproton lab kinetic energy for the impact parameter value $b=10^{10} \mathrm{fm}$. In the upper panel the results in PW are divided by 1000. In the lower panel the PW results

Fig. 4. Absolute value of the integrated polarisation transfer cross-section $\left|\left\langle P_{z z} \sigma\right\rangle\right|$ for proton electron scattering in the c.m. function of the antiproton lab kinetic energy for $b=10^{10} \mathrm{fm}$. are divided by 100 . 